\title{
Editorial
}

\section{Nutrigenomics and Systems Biology}

Jing X. Kang

Massachusetts General Hospital and Harvard Medical School, Boston, Mass., USA

Systems biology has emerged as a critical concept for biological and medical research. Its growing recognition stems from the reality that organisms exist as systems, and the better we understand the interactions in these complex biological networks, the more readily we can develop effective prevention or treatment strategies that comprehensively address health problems. The field of systems biology therefore offers us a unique perspective in both theory and practice. At its core, systems biology emphasizes the interactions between biological mechanisms and how these signaling networks yield emergent properties of the system as a whole. In research, the implementation of this concept warrants the integration of systemwide experimental techniques to gather a complete bank of data on the numerous processes within a system. With today's cutting-edge 'omics' technologies - including transcriptomics, proteomics, metabolomics, and lipidomics - we are capable of not just analyzing components of interest, but also constructing quantitative, verifiable models of biological systems to see how altering those components may affect a system overall. As the power of systems biology evolves, we must utilize its principles in the study of nutrigenomics as well as in translating the results into clinical applications.

From a biological standpoint, nutrigenomics is actually an important domain of systems biology, from its research concept to its practical application. Nutrigenomics mainly deals with the interactions between nutrients and genes. On a larger scale, nutrigenomics reflects the interaction between an environmental factor (nutrients) and our biological systems (genes). Before we can evaluate the nutrient-gene interactions, we must also take into account the systemic factors that affect the bioavailability and tissue concentration of the nutrients of interest, including digestion, absorption, and transportation. At the molecular level, the evaluation of nutrigenomic effects requires a thorough examination of at least three interconnected systems: nutrient-nutrient, nutrient-gene, and gene-gene interactions. In terms of nutrient-nutrient interactions, one type of nutrient can significantly affect the metabolism and biological function of another nutrient. For example, omega- 6 and omega- 3 polyunsaturated fatty acids are both essential fatty acids, but they compete with each other for metabolic 
enzymes and incorporation into cell membranes, and differentially affect gene expression [1]. In nutrient-gene interactions, the same nutrient can modulate gene expression in different ways. For example, essential fatty acids can directly bind to transcriptional factors, such as peroxisome proliferator-activated receptors (PPARs), to regulate lipid metabolism genes, but their metabolites, such as eicosanoids, can also bind to their receptors to signal gene expression [2]. In gene-gene interactions, one gene change can alter many other related gene expressions. For example, activation of nuclear factor kappa B (NFkB) can induce the expression of various cytokines, which can in turn upregulate the activity of NFкB [3]. In brief, one nutrient can affect multiple gene expressions, and one gene expression can be modulated by different nutrients. As we can see that the complex relationship between nutrients and gene expression cannot and should not be reduced to individual interactions, it therefore follows that the field of nutrigenomics is a prime subject for the application of systems biology.

From a practical standpoint, the integrated approach of systems biology can inspire us to create comprehensive strategies for more effective prevention and treatment of diseases. Current medicine is largely based on the 'one drug, one target, one disease' model; while this sounds ideal in theory, many of the existing drugs exhibit limited efficacy with unwanted side effects. One major clinical condition of interest is inflammation, as its chronic state can cause and promote many serious diseases, such as cardiovascular disease, obesity, diabetes, and cancer [4]. The inflammatory response involves multiple biological processes, including lipid mediator generation, immune cell activation and migration, cytokine secretion, and oxidative stress formation. Most anti-inflammatory drugs only target a specific enzyme or pathway, such as COX-2 inhibitors; in many cases, the outcome with these drugs is not quite satisfactory with additional side effects, and they usually are not safe for long-term, preventive purposes. A nutrigenomic approach guided by systems biology principles may provide alternative interventions for this condition. For example, by identifying the nutrients that are particularly effective in modulating certain steps of inflammation, we are capable of utilizing specific nutrient combinations to target multiple pathways and thereby suppress inflammation in a widely effective, non-toxic manner. The development of such formulas may be the missing key to safe and reliable treatment strategies for inflammation and other related health conditions.

With the principles of systems biology in mind, we should recognize the complexity of biological systems and utilize an integrated approach to investigate the interactions between nutrients and genes at all potential levels. By doing so, we will be able to obtain reliable and comprehensive information for the development of personalized nutrition.

\section{References}

Weylandt KH, Kang JX: Rethinking lipid mediators. Lancet 2005;366:618-620.

Funk CD: Prostaglandins and leukotrienes: advances in eicosanoid biology. Science 2001;294:1871-1875.

Kang JX, Weylandt KH: Modulation of inflammatory cytokines by omega-3 fatty acids. Subcell Biochem 2008; 49:133-143.

4 Hotamisligil GS: Inflammation and metabolic disorders. Nature 2006;444:860-867. 